\title{
Perfil e distribuição espacial da sífilis congênita em Sobral-CE no período de 2007 a 2013
}

\author{
Profile and space distribution of congenital syphilis in Sobral-CE between 2007 and 2013
}

\author{
Ilana Marques Rodriguesa, Marcos Aguiar Ribeirob, Izabelle Mont'Alverne Napoleão Albuquerquec, \\ Lívia Karla Sales Dias ${ }^{d}$, Nara Luana Trajano Aguiare, Danyella dos Santos Lima \\ a Enfermeira. Especialista em Saúde do Trabalhador. Membro do Observatório de Pesquisas para o SUS. \\ benfermeiro. Especialista em Gestão da Saúde e Auditoria. Mestrando em Saúde da Família pela Universidade Federal do Ceará (UFC). \\ Docente do curso de Enfermagem da Universidade Estadual Vale do Acaraú. Membro do Observatório de Pesquisas para o SUS. \\ Enfermeira. Doutora em Enfermagem pela UFC. Docente da Universidade Estadual Vale do Acaraú. Docente do Mestrado Acadêmico em Saúde da Família da UFC. \\ Vice-reitora e docente do Mestrado Profissional em Saúde da Família da Rede Nordeste de Formação em Saúde da Família (RENASF/FIOCRUZ). \\ Lider do Observatório de Pesquisas para o SUS \\ Enfermeira. Mestre em Saúde da Família pela UFC. Membro do Observatório de Pesquisas para o SUS. \\ Acadêmica do Curso de Enfermagem pela Universidade Estadual Vale do Acaraú. Membro do Observatório de Pesquisas para o SUS. \\ Bolsista de Iniciação Científica pelo Conselho Nacional de Desenvolvimento Científico e Tecnológico (CNPq). \\ Enfermeira graduada pela Universidade Estadual Vale do Acaraú. Membro do Observatório de Pesquisas para o SUS.
}

RESUMO

Objetivo: Analisar a distribuição espacial da sífilis congênita no Sistema Municipal de Saúde de Sobral/CE, utilizando técnicas de geoprocessamento.

Materiais e Métodos: Estudo de abordagem quantitativa do tipo epidemiológico-ecológico-transversal com dados do Sistema de Informação de Agravos de Notificação, sobre sífilis congênita no período de 2007 a 2013. Para o processamento das informações foi utilizado o software Epilnfo ${ }^{\circledR}$ que possibilitou as tabulações e mapeamento dos dados coletados cuja análise se deu por meio de medidas de frequências.

Resultados: Evidencia-se um considerável aumento do percentual dos casos de sífilis congênita no período do estudo apesar da crescente cobertura do Pré-Natal. No que se refere a distribuição espacial, comprova-se por meio dos mapas que nas regiões onde as condições socioeconômicas da população são desfavoráveis ocorrem aglomerados de casos de sífilis congênita. Estas regiões estão localizadas predominantemente nas periferias da cidade.

Conclusão: A partir do estudo revelam-se alguns pontos frágeis da assistência e prevenção da sífilis, tais como a investigação inadequada dos casos de sífilis na gravidez, o tratamento inadequado da gestante e a não realização do tratamento do parceiro. Assim, o geoprocessamento da sífilis congênita é uma ferramenta tecnológica relevante, pois contribui para a prevenção, vigilância e controle da mesma, de forma que possibilita análise dos determinantes envolvidos no processo saúde-doença.

Palavras-chave: sífilis congênita; sistemas de informação geográfica; saúde coletiva.

\section{ABSTRACT}

\begin{abstract}
Objective: To analyze the spatial distribution of congenital syphilis in the Municipal System of Health of Sobral - CE, using geoprocessing techniques.

Material and Methods: A quantitative epidemiological-ecological cross-sectional study with information from the Aggravated Syphilis Information System on congenital syphilis from 2007 to 2013 was used. Epilnfo ${ }^{\circledR}$ software was used to process the information. Tabulations and mapping of the collected data that were analyzed by means of frequency measurements.

Results: There was a considerable increase in the percentage of congenital syphilis cases in the study period despite the increase in prenatal coverage. As regards the spatial distribution, the maps demonstrated that in regions where the socioeconomic conditions of the population are unfavorable, agglomerates of congenital syphilis cases occur. These regions are located predominantly in the outskirts of the city.

Conclusion: The study reveals some fragile aspects of syphilis care and prevention, such as inadequate investigation of syphilis in pregnancy, inadequate treatment of the pregnant woman and failure to perform treatment of the partner. Thus, the geoprocessing of congenital syphilis is a relevant technological tool, since it contributes to the prevention, surveillance and control of it, so that it allows the analysis of the determinants involved in the health-disease process.
\end{abstract}




\section{INTRODUÇÃO}

Para a gestão organizada dos serviços de saúde é necessária a sistematização de informações, pois os sistemas de saúde e a sua prestação de serviços tem levado a uma crescente demanda por informações que exigem o gerenciamento de recursos públicos. Nessa perspectiva, a utilização de técnicas de análise espacial por meio do geoprocessamento tem despertado muito interesse ao setor saúde, uma vez que permite ter uma visão abrangente da saúde dos indivíduos no contexto social, político, cultural e ambiental em que estão inseridos.

Nesse sentido, o geoprocessamento pode ser definido como um Sistema de Informação Geográfica (SIG), que utiliza um conjunto de técnicas computacionais necessárias para manipular informações espacialmente referidas. Aplicado à Saúde Coletiva permite o mapeamento de doenças, a avaliação de riscos, o planejamento de ações de saúde e a avaliação de redes de atenção ${ }^{1}$.

O geoprocessamento é considerado uma ferramenta bastante eficaz que possibilita aos gestores uma visualização estrutural e contextualizada, favorecendo a síntese/integração de variáveis que justificam a tomada de decisões, pois todas estas informações estão geograficamente distribuídas pelo território.

A sífilis, conhecida desde o século XV, é uma enfermidade sistêmica causada pelo Treponema pallidum, exclusiva do ser humano e tem como principal via de transmissão o contato sexual, seguido pela transmissão vertical para o feto durante o período de gestação de uma mãe com sífilis não tratada ou tratada inadequadamente. Também pode ser transmitida por transfusão sanguínea. A apresentação dos sinais e sintomas da doença é muito variável e complexa. Quando não tratada, evolui para formas mais graves, podendo comprometer o sistema nervoso, o aparelho cardiovascular, o aparelho respiratório e o aparelho gastrointestinal ${ }^{2}$.

A Organização Mundial de Saúde $(\mathrm{OMS})^{3}$ estima que no mundo todo ocorra um milhão de casos de sífilis por ano entre as gestantes e preconiza a detecção e o tratamento oportunos destas e de seus parceiros sexuais portadores da sífilis, considerando que a infecção pode ser transmitida ao feto, com graves implicações.

Neste contexto, no ano de 2016, foram notificados no Brasil 87.593 casos de sífilis adquirida, 37.436 casos de sífilis em gestantes e 20.474 casos de sífilis congênita, o que impactou em 185 óbitos. No que se refere a sífilis congênita, verifica-se uma elevação das taxas de incidência, que aumentaram cerca de três vezes, passando de 2,4 em 2010 para 6,8 em $2016^{4}$.

Assim, o estudo tem como objetivo analisar a distribuição espacial da sífilis congênita no Sistema Municipal de Saúde de Sobral/CE, utilizando técnicas de geoprocessamento.

\section{MATERIAIS E MÉTODOS}

Trata-se de um estudo de abordagem quantitativa, classificado como epidemiológico, de referência temporal transversal.

O campo de investigação foi o Sistema Municipal de Saúde de Sobral através do recorte dos territórios de Saúde da Família da Sede do município. A atenção primária à saúde (APS) Sobral conta atualmente com 63 equipes de Estratégia de Saúde da Família (ESF), 37 equipes e saúde bucal, 07 equipes de Núcleo de Apoio à Saúde da Família (NASF) distribuídas em 33 Centros de Saúde da Família (CSF).

O critério da acessibilidade permeou a escolha do recorte do campo de estudo para os territórios de Saúde da Família da Sede e da Zona Rural do referido município. A Sífilis congênita atendeu o critério de elegibilidade, pois se configura como doença de notificação compulsória no território brasileiro 5 .

A população do estudo foram 120 recém-nascidos (RN) de parto cesariano e vaginal, nascidos nos dois hospitais de referência no referido município com diagnóstico de Sífilis Congênita, confirmados no período de 2007 a 2013 desde que assistidos pela Estratégia Saúde da Família dos territórios de Sobral-CE, sendo suas informações acessadas por meio de fontes documentais.

Assim, a busca da captação dos conteúdos e informações acerca do objeto em estudo nos levou a eleger a consulta a fontes documentais como método de coleta de dados. Desse modo, foram utilizadas as informações do Sistema Nacional de Agravos de Notificação (SINAN). Foram extraídos os arquivos do SINAN referentes à Sífilis Congênita para o período de janeiro de 2007 a dezembro de 2013 totalizando uma amostra 120 notificações de RN com a confirmação da doença.

Através dos estudos das informações contidas no Sistema de Vigilância do Serviço de saúde de Sobral/CE constituíram-se como variáveis do estudo: Os antecedentes epidemiológicos da gestante, os dados laboratoriais do RN, dados do diagnóstico e da notificação e evolução da doença.

Os dados extraídos do SINAN foram processados nas seguintes etapas: tabulação, realização de testes estatísticos simples e mapeamento, etapas desenvolvidas através da utilização do software Epilnfo versão 7.0 (Centers for Disease Controland Prevention, Atlanta, Estados Unidos). Destaca-se ainda que o endereço de residência foi a unidade de análise para o georreferenciamento dos dados. Posteriormente, foram obtidas as frequências das variáveis estudadas. Para apresentação dos dados optou-se pela construção de gráficos, tabelas e mapas. 
Vale salientar que a presente pesquisa obteve parecer favorável do Comitê de Ética em Pesquisa da Universidade Estadual Vale do Acaraú, com número de parecer: 470.655, sendo orientado a partir da Resolução de no 466 de 12 de dezembro de 2012 do Conselho Nacional de Saúde ${ }^{6}$.

\section{RESULTADOS}

No período referente aos anos de 2007 a 2013 foram notificados nos territórios da Estratégia Saúde da Família (ESF) do município de Sobral/CE 120 casos de Sífilis Congênita. Estes números possuem uma curva ascendente o que nos possibilita inferir que as medidas adotadas necessitam serem avaliadas, especialmente no que concerne à busca ativa e tratamento de parceiros. A citar que no ano de 2007 foram notificados cinco casos de Sífilis Congênita, enquanto que no ano 2013 este número cresceu para 16, ou seja, um aumento considerável de $68,7 \%$.

A pesquisa contemplou diversas variáveis, que por vez foram agrupadas e estão relacionadas ao RN, que concernem aos dados laboratoriais dos exames dos mesmos, sendo importante verificar os percentuais e frequências, que estão apresentados na Tabela $\mathbf{1}$.

Coube ainda destacar os fatores sócio-demográficos e antecedentes epidemiológicos da gestante/mãe associados ao diagnóstico da sífilis gestacional, pois contribuem para uma melhor compreensão acerca dos resultados obtidos nas variáveis relacionadas diretamente a criança com diagnóstico de sífilis congênita, sendo estas informações contidas na Tabela 2.

O mapa a seguir (Figura 1) mostra que durante o período de sete (07) anos consecutivos os casos de Sífilis Congênita estão centralizados nos bairros onde tem concentração de população de alta vulnerabilidade social. Verifica-se que o geoprocessamento é uma tecnologia que pode ser aplicada ao trabalho dos profissionais da ESF. Dessa forma, os dados foram georreferenciados, de forma a apresentar a distribuição espacial da Sífilis Congênita em Sobral. A Figura 2 apresenta os casos de Sífilis Congênita no período de 2007 a 2013.

A partir do mapa (Figura 1) podem-se observar aglomerados de casos nos bairros situados na periferia da cidade, regiões estas, marcadas por vulnerabilidades sociais, como baixos índices socioeconômicos e grandes aglomerados populacionais. Quanto as Figuras 2 e $\mathbf{3}$ apresentam respectivamente a distribuição espacial dos casos de Sífilis Congênita estratificados por parceiros que não foram tratados e Tratamento Inadequado da gestante, ambos referentes ao período de 2007 a 2013.
Tabela 1. Dados laboratoriais da criança com sífilis congênita.

\begin{tabular}{|c|c|c|}
\hline Variáveis & n & Percentual \\
\hline \multicolumn{3}{|l|}{ Sexo } \\
\hline Masculino & 48 & 51,05 \\
\hline Feminino & 70 & 48,95 \\
\hline Ignorado & 2 & 1,67 \\
\hline \multicolumn{3}{|c|}{ Teste não treponêmico - Sangue periférico } \\
\hline Reagente & 80 & 66,67 \\
\hline Não reagente & 27 & 22,50 \\
\hline Não realizado & 12 & 10,00 \\
\hline Ignorado & 1 & 0,83 \\
\hline \multicolumn{3}{|c|}{ Teste treponêmico ( após 18 meses) } \\
\hline Reagente & 0 & 0,00 \\
\hline Não reagente & 5 & 4,17 \\
\hline Não realizado & 59 & 49,17 \\
\hline Não se aplica & 43 & 35,83 \\
\hline Ignorado & 13 & 10,83 \\
\hline \multicolumn{3}{|l|}{ Teste não treponêmico (Líquor) } \\
\hline Reagente & 1 & 0,83 \\
\hline Não reagente & 26 & 21,67 \\
\hline Não realizado & 78 & 65,00 \\
\hline Ignorado & 15 & 12,50 \\
\hline \multicolumn{3}{|c|}{$\begin{array}{l}\text { Titulação ascendente } \\
\text { (VDRL com 01,03, 06,12 e } 18 \text { meses) }\end{array}$} \\
\hline Sim & 2 & 1,67 \\
\hline Não & 2 & 1,67 \\
\hline Não realizado & 82 & 68,33 \\
\hline Ignorado & 34 & 28,33 \\
\hline \multicolumn{3}{|c|}{ Evidência de treponema pallidum } \\
\hline Sim & 3 & 2,50 \\
\hline Não & 9 & 7,50 \\
\hline Não realizado & 78 & 65,00 \\
\hline Ignorado & 30 & 25,00 \\
\hline \multicolumn{3}{|l|}{ Alteração liquórica } \\
\hline Sim & 0 & 0,00 \\
\hline Não & 32 & 26,67 \\
\hline Não realizado & 70 & 58,33 \\
\hline Ignorado & 18 & 15,00 \\
\hline \multicolumn{3}{|l|}{ Diagnóstico Clínico } \\
\hline Assintomático & 95 & 79,17 \\
\hline Sintomático & 6 & 5,00 \\
\hline Não se aplica & 7 & 5,83 \\
\hline Ignorado & 12 & 10,00 \\
\hline \multicolumn{3}{|c|}{$\begin{array}{l}\text { Diagnóstico radiológico da criança: } \\
\text { alteração do exame dos ossos longos }\end{array}$} \\
\hline Sim & 3 & 2,50 \\
\hline Não & 32 & 26,57 \\
\hline Não realizado & 77 & 64,17 \\
\hline Ignorado & 8 & 6,67 \\
\hline \multicolumn{3}{|l|}{ Evolução do caso } \\
\hline Vivo & 115 & 95,83 \\
\hline Óbito por sífilis congênita & 1 & 0,83 \\
\hline Aborto & 3 & 2,50 \\
\hline Ignorado & 1 & 0,83 \\
\hline
\end{tabular}


Tabela 2. Fatores sociodemográficos e antecedentes epidemiológicos gestante/mãe.

\begin{tabular}{|c|c|c|}
\hline Variáveis & $\mathbf{n}$ & Percentual \\
\hline \multicolumn{3}{|l|}{ Idade da Mãe (anos) } \\
\hline Média \pm dp & $26,75 \pm 5,66$ & \\
\hline Moda & 21 & \\
\hline Mediana & 25 & \\
\hline \multicolumn{3}{|l|}{ Raça/Cor da Mãe } \\
\hline Branca & 6 & 5,00 \\
\hline Preta & 2 & 1,67 \\
\hline Amarela & 1 & 0,83 \\
\hline Parda & 111 & 92,50 \\
\hline \multicolumn{3}{|l|}{ Escolaridade da Mãe } \\
\hline Analfabeto & 1 & 0,83 \\
\hline 1a a 4 a série incompleta - ensino fundamental & 8 & 6,67 \\
\hline 4⿳a série completa - ensino fundamental & 2 & 1,67 \\
\hline 5 a a $8^{\underline{a}}$ série incompleta - ensino fundamental & 43 & 35,83 \\
\hline Ensino fundamental completo & 10 & 8,33 \\
\hline Ensino médio incompleto & 10 & 8,33 \\
\hline Ensino médio completo & 9 & 7,50 \\
\hline Educação superior incompleta & 0 & 0,00 \\
\hline Educação superior completa & 1 & 0,83 \\
\hline Ignorado & 35 & 29,17 \\
\hline Não se aplica & 1 & 0,83 \\
\hline \multicolumn{3}{|l|}{ Realização do Pré-Natal } \\
\hline Sim & 116 & 96,67 \\
\hline Não & 3 & 2,50 \\
\hline Ignorado & 1 & 0,83 \\
\hline \multicolumn{3}{|l|}{ Diagnóstico da Sífilis materna } \\
\hline Durante o Pré- Natal & 116 & 96,67 \\
\hline No momento do parto/ curetagem & 1 & 0,83 \\
\hline Pós parto & 2 & 1,67 \\
\hline Ignorado & 1 & 0,83 \\
\hline \multicolumn{3}{|l|}{ Esquema de Tratamento da gestante } \\
\hline Adequado & 8 & 6,67 \\
\hline Inadequado & 97 & 80,83 \\
\hline Não Realizado & 12 & 10,00 \\
\hline Ignorado & 3 & $2,, 50$ \\
\hline \multicolumn{3}{|l|}{$\begin{array}{l}\text { Parceiro tratado concomitantemente } \\
\text { a gestante }\end{array}$} \\
\hline Sim & 16 & 13,33 \\
\hline Não & 94 & 78,33 \\
\hline Ignorado & 10 & 8,33 \\
\hline \multicolumn{3}{|l|}{$\begin{array}{l}\text { Teste confirmatório treponêmico no } \\
\text { parto/curetagem }\end{array}$} \\
\hline Não realizado & 115 & 95,83 \\
\hline Reagente & 1 & 0,83 \\
\hline Não reagente & 1 & 0,83 \\
\hline Ignorado & 3 & 2,50 \\
\hline \multicolumn{3}{|l|}{ Hospital do Parto/ Curetagem } \\
\hline Hospital Dr. Estevam & 41 & 34,17 \\
\hline Santa Casa de Misericórdia de Sobral & 72 & 60,00 \\
\hline Outro & 7 & 5,83 \\
\hline \multicolumn{3}{|l|}{$\begin{array}{l}\text { Teste confirmatório não treponêmico no } \\
\text { parto/curetagem }\end{array}$} \\
\hline Não realizado & 4 & 1,66 \\
\hline Reagente & 114 & 95,00 \\
\hline Não reagente & 1 & 0,83 \\
\hline Ignorado & 1 & 0,83 \\
\hline
\end{tabular}

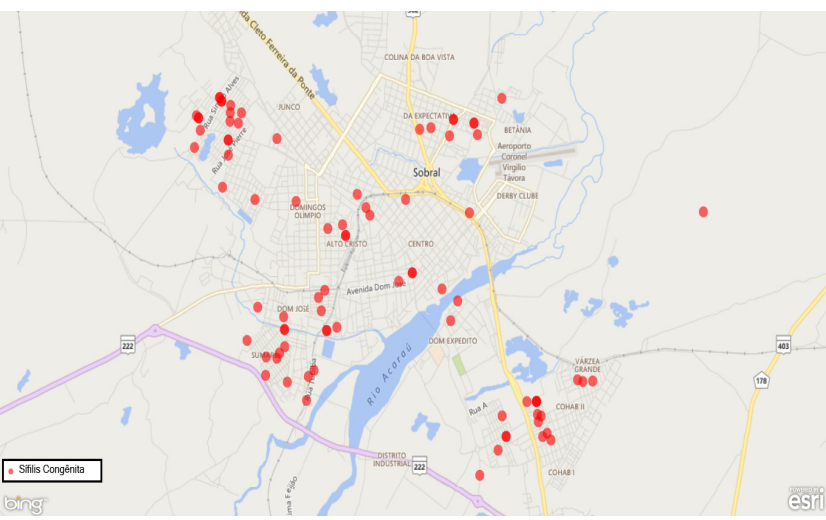

Figura 1. Georreferenciamento dos casos de Sífilis Congênita referente ao período de 2007 a 2013.

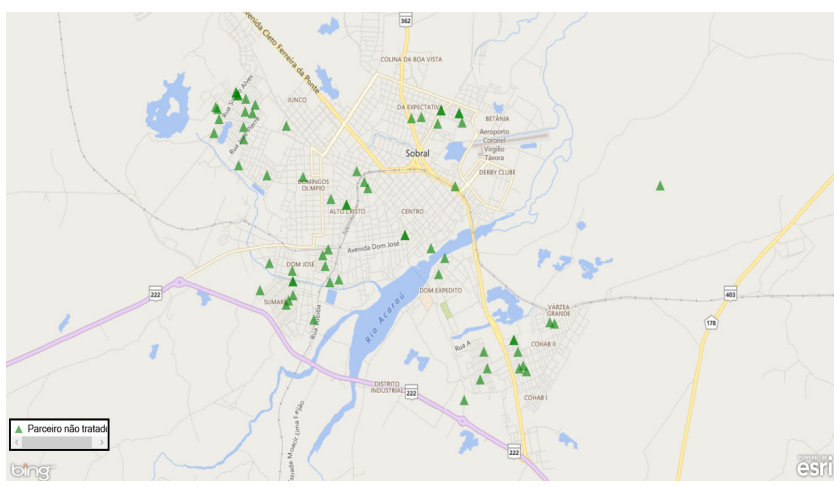

Figura 2. Georreferenciamento dos casos de Sífilis Congênita estratificados por parceiros que não foram tratados, referente ao período de 2007 a 2013.

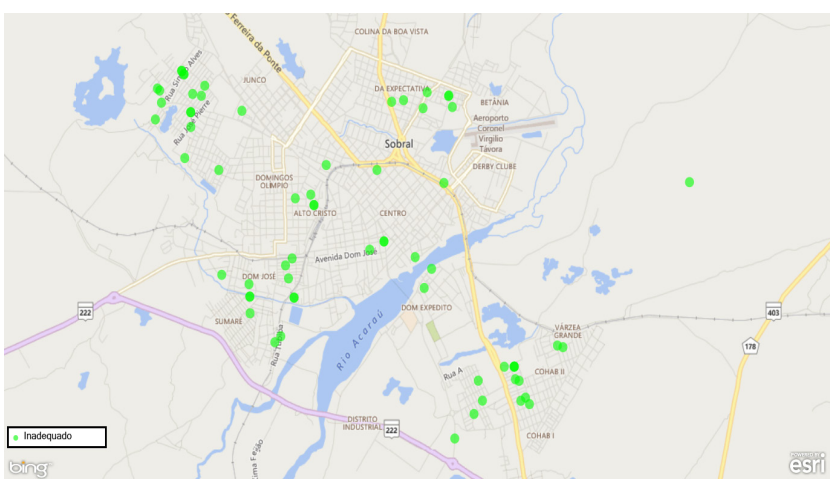

Figura 3. Georreferenciamento dos casos de Sífilis Congênita estratificado por Tratamento Inadequado da gestante, referente ao período de 2007 a 2013.

A partir do gráfico ilustrado a seguir (Figura 4 ) evidencia o considerável aumento do percentual dos casos de Sífilis Congênita no período de 2007 a 2013, saindo de 2,5\% para $35 \%$ em sete anos. Em contrapartida os resultados deste estudo comprovam que a cobertura do Pré-Natal (PN), realizados pelas equipes das ESF no municipio de Sobral, referente ao mesmo período foi de $96,67 \%$. 


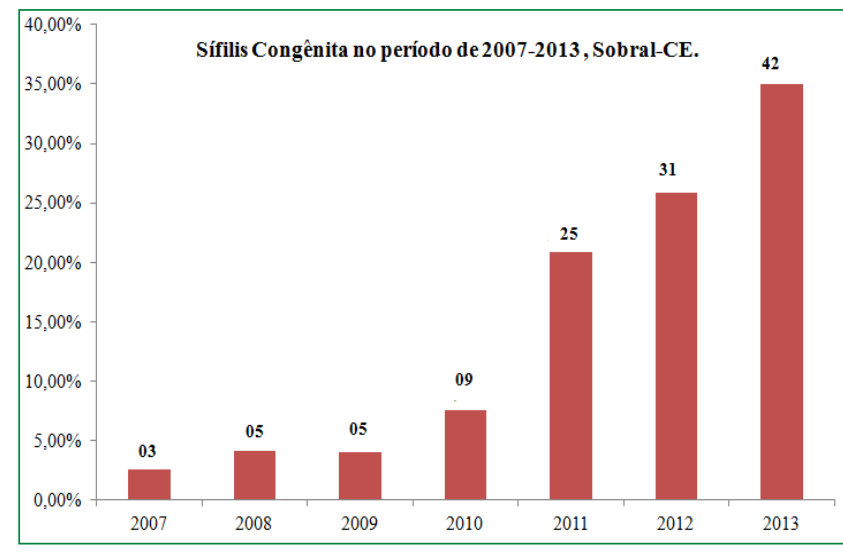

Figura 4. Percentual dos casos de Sífilis Congênita notificados entre 2007 e 2013.

\section{DISCUSSÃO}

O município de Sobral - CE intensificou a vigilância da sífilis em gestante somente a partir 2006 e da sífilis adquirida em 2008. Este monitoramento tardio da sífilis adquirida pode ter resultado em subnotificações ou sub-registro da doença na população do sexo masculino, que pode ter contribuído para a elevação de casos em gestantes nos últimos anos e sua consequente transmissão vertical ${ }^{7}$.

O aumento do número de casos de sífilis congênita notificados no período de 2007 a 2013, em Sobral demonstra a necessidade do desenvolvimento de ações efetivas voltadas a seu controle, assim como de educação em saúde para a população, uma vez que tratar-se de uma doença totalmente evitável desde que seja feito o diagnóstico precoce e estabelecido tratamento adequado para a gestante infectada e seu(s) parceiro(s). Nesse sentido, é importante o fortalecimento da qualificação da atenção à saúde por meio do compartilhamento de responsabilidades, comunicação em saúde, educação permanente em saúde, qualificação das informações estratégicas e fortalecimento da parceria com outros atores e ampliação dos comitês de investigação ${ }^{4}$.

O Boletim Epidemiológico de Sífilis divulgado em 2015 pelo Ministério da Saúde permite uma visão panorâmica da realidade Nacional no que se refere ao número de casos de sífilis. De acordo com este documento, de 1998 a junho de 2014 foram notificados no SINAN 104.853 casos de sífilis congênita em menores de um ano de idade, dos quais 32.884 $(31,4 \%)$ foi na região Nordeste. Com relação à incidência de sífilis congênita, em 2013 observou se uma taxa de 4,7 casos por 1.000 nascidos vivos no Brasil, sendo que a Região Nordeste apresentou a maior incidência de casos $(5,3)$, seguida da Sudeste $(5,1)$, Sul $(4,1)$, Norte $(3,5)$ e CentroOeste $(3,3)^{8}$. Assim, nota-se que o cenário vislumbrado no município de Sobral corrobora com a realidade brasileira, em especial região Nordeste.

Vale destacar que apesar dos índices elevados de sífilis é preciso considerar ainda a subnotificação da sífilis congênita. A notificação da sífilis na gestação é obrigatória desde 2005 e é marcada por elevadas taxas de prevalência e de transmissão vertical. A sífilis congênita passou a ser de notificação compulsória em 1986 e sua ficha de investigação sofreu variações ao longo dos anos, a fim de melhorar a detecção e acompanhar o processo de eliminação assinado em acordos internacionais pelo Ministério da Saúde ${ }^{9}$.

Diante da realidade nacional a política de prevenção da morbimortalidade materno-infantil do Pacto pela Saúde do Ministério da Saúde (2006) incluiu metas de redução da transmissão vertical da sífilis contidas no Plano Plurianual (PPA) 2012-2015 ${ }^{10}$. Em 2011, o Governo Federal lançou a "Rede Cegonha". Uma das suas estratégias é a implantação dos testes rápidos diagnóstico do HIV e triagem da sífilis no âmbito da Atenção Básica no intuito de promover um diagnóstico precoce desses agravos nas gestantes e o início oportuno das ações de prevenção, com vistas à eliminação da sífilis congênita, bem como à redução de óbitos maternoinfantis evitáveis ${ }^{11}$. Com a expansão do diagnóstico por meio de testes rápidos de sífilis, observou-se elevação na taxa de detecção de sífilis em gestantes, decorrente da melhoria do diagnóstico e da vigilância epidemiológica ${ }^{12}$.

Relevante destacar que o estudo do nível de escolaridade se configura como importante ferramenta de análise da população estudada, uma vez que se pode considerar a associação entre a baixa escolaridade e a dificuldade no acesso aos serviços de saúde e na promoção da saúde e prevenção de doenças ${ }^{13}$. Entretanto, outro fator preocupante foi à ocorrência de casos de sífilis congênita em RN de mães com 12 ou mais anos de escolaridade, pois se espera que essa população tenha conhecimentos mínimos acerca das Doenças Sexualmente Transmissíveis (DST) e suas formas de prevenção, além da importância da realização do PN.

À semelhança de outros estudos ${ }^{13,14}$, a maior parte das gestantes realizou acompanhamento pré-natal. Apesar do número ascendente de pré-natal realizado durante os anos, verificou-se um percentual crescente do número de casos de sífilis congênita. Esses dados podem revelar tanto a qualidade da assistência pré-natal, que permanece aquém da desejada, quanto o aumento dos casos notificados que, mesmo sendo ainda inferior ao esperado, vem mostrando avanços ${ }^{4}$.

Além disso, cabe destacar que não basta ampliar a cobertura dos testes sorológicos de sífilis durante o PN, é necessário que os resultados estejam disponibilizados em tempo hábil, para que os profissionais possam implementar medidas preventivas da transmissão vertical ${ }^{9}$. 
Indubitavelmente, a existência de casos de sífilis congênita em municípios com altas coberturas de ESF, como é o caso de Sobral/CE, sugere problemas no funcionamento de suas equipes e/ou na organização do sistema de saúde, como a garantia de acesso a exames laboratoriais.

O presente estudo apresenta um expressivo percentual de tratamento inadequado da gestante com sífilis. Este achado é convergente com um estudo realizado no Pará com 46 casos de sífilis congênita, somente $53,8 \%$ das mães que tiveram o diagnóstico de sífilis durante o pré-natal receberam e tratamento adequado ${ }^{15}$. Em um estudo similar realizado em São Paulo com 45 mães de recém-nascidos com diagnóstico de sífilis congênita identificou-se que em menos de 50\% dos casos, o tratamento da mãe, antes e após o parto foi registrado como adequado ${ }^{16}$.

Outro ponto a ressaltar é que conforme o mapeamento dos casos onde há maior número de gestantes tratadas inadequadamente também se encontra presente a maior concentração de homens não tratados. Ressalta-se que o tratamento adequado da sífilis na gestação é considerado toda a terapêutica de acordo com o estágio da doença, e finalizado pelo menos 30 dias antes do parto, tendo sido o parceiro tratado concomitantemente ${ }^{16}$.

Os dados do Boletim Epidemiológico da Sífilis ${ }^{4}$ convergem com os achados do estudo, uma vez que verificase em âmbito nacional que $62,2 \%$ dos casos o parceiro não foi tratado, $15,2 \%$ fizeram tratamento e para $22,6 \%$ dos casos essa informação é ignorada/em branco. Isso significa que o enfrentamento da sífilis não depende apenas de conhecimentos biomédicos, recursos humanos e materiais, pois envolve intervenções não somente sobre aspectos biológicos, mas também comportamentais e socioculturais ${ }^{17}$.

Com relação à distribuição por faixa etária, observou-se um predomínio de mulheres de 21 a 29 anos. Dessa forma, vale destacar que apesar da adolescência ser fator de risco para sífilis, dentre as mães com a infecção apenas um pequeno quantitativo eram adolescentes. Estes achados corroboram com os dados do Ministério da Saúde ${ }^{4}$, que evidencia no Brasil para o ano de 2017 que as maiores proporções de casos de sífilis congênita ocorreram em crianças cujas mães tinham entre 20 e 29 anos de idade (54,1\%).

Além disso, a titulação ascendente é essencial para a comparação dos títulos da sorologia não treponêmica da criança após cada teste realizado durante o esquema de seguimento, conforme preconiza o Ministério da Saúde ${ }^{8}$. A partir de então, pode-se suscitar, a partir do considerável número de casos onde não foram realizados esses exames, que existem limitações no acompanhamento dos casos por meio dos exames ou as informações não são retroalimentadas no SINAN.
Os achados deste estudo revelam alguns pontos frágeis da assistência e prevenção da sífilis: a investigação inadequada dos casos de sífilis na gravidez; o tratamento inadequado da gestante e a não realização do tratamento do parceiro. É necessária a capacitação das equipes da ESF, as quais, certamente, têm muito a colaborar na promoção de mudanças no quadro da doença, visto tratarse da porta de entrada do sistema de saúde, possibilitando maior proximidade entre os profissionais e os usuários, especialmente os parceiros de gestantes infectadas, para conseguir sua adesão ao tratamento.

Neste contexto, infere-se que o geoprocessamento é uma tecnologia que pode ser aplicada ao trabalho dos profissionais da saúde. Configura-se como uma importante ferramenta tecnológica de trabalho, pois possibilita a visualização espacial dos casos de sífilis congênita em todo território de abrangência da ESF do município de Sobral o que poderá contribuir para o planejamento e avaliação das ações de saúde desenvolvidas no município acerca da doença. Assim, pode-se considerar a utilização desta tecnologia como mais um instrumento de gestão da vigilância em saúde.

\section{AGRADECIMENTO}

Agradecemos ao Conselho Nacional de Desenvolvimento Científico e Tecnológico (CNPq) pela concessão da bolsa de Iniciação Científica.

\section{REFERÊNCIAS}

1. Ministério da Saúde (BR). Secretaria de Vigilância em Saúde. Departamento de Vigilância Epidemiológica. Sistema de Informação de Agravos de Notificação - Sinan: normas e rotinas. Brasília (DF); 2007.

2. Ministério da Saúde (BR). Sífilis: Estratégias para Diagnóstico no Brasil. Coordenação de Doenças Sexualmente Transmissíveis e Aids. Brasília (DF); 2010.

3. Organizacion Mundial da Saúde. Orientaciones mundiales sobre los criterios y procesos para la validacion de la eliminacion de la transmision maternoinfantil del VIH y la sifilis. Ginebra; 2015.

4. Ministério da Saúde (BR). Secretaria de Vigilância em Saúde. Boletim Epidemiológico da Sífilis 2017. Brasília (DF); 2017.

5. Ministério da Saúde (BR). Portaria no 204, de 17 de fevereiro de 2016. Define a Lista Nacional de Notificação Compulsória de doenças, agravos e eventos de saúde pública nos serviços de saúde públicos e privados em todo o território nacional, nos termos do anexo, e dá outras providências. D.O. República Federativa do Brasil. 2016;18(32):23.

6. Ministério da Saúde (BR). Conselho Nacional de saúde. Resolução de no 466, de 12 de dezembro de 2012. D O República Federativa do Brasil. 2012;13(1):59.

7. Mesquita KO, Lima GK, Filgueira AA, Flôr, SMC, Freitas CASL, Linhares MSC, Gubert FA. Análise dos casos de sífilis congênita em Sobral, Ceará: contribuições para assistência pré-natal. DST J Bras Doenças Sex Transm. 2012;24(1):20-7. https://doi. org/10.5533/2177-8264-201224107 
8. Ministério da Saúde (BR). Secretaria de Vigilância em Saúde. Boletim Epidemiológico de Sífilis, ano IV, n. 1. Brasília (DF): Ministério da Saúde; 2015.

9. Ministério da Saúde (BR). Secretaria de Vigilância em Saúde. Programa Nacional de DST e Aids. Diretrizes para o Controle da Sífilis Congênita/Ministério da Saúde, Secretaria de Vigilância em Saúde, Programa Nacional de DST e Aids. Brasília (DF): Ministério da Saúde; 2005.

10. Ministério da Saúde (BR). Secretaria-Executiva. Departamento de Apoio à Descentralização. Regulamento dos pactos pela vida e de gestão. Brasília (DF): Ministério da Saúde; 2006.

11. Cavalcanti PCS, Gurgel JGD, Vaconcelos ALR, Gerrero AVP. Um modelo lógico da Rede Cegonha. Physis. 2013;23(4):1297-316. https://doi.org/10.1590/S0103-73312013000400014

12. Saraceni V, Miranda AE. Relação entre a cobertura da Estratégia Saúde da Família e o diagnóstico de sífilis na gestação e sífilis congênita. Cad Saúde Pública. 2012;28(3):490-46. https://doi. org/10.1590/S0102-311X2012000300009
13. Ximenes IPE, Moura ERF, Freitas GL, Oliveira NC. Incidência e controle da sífilis congênita no Ceará. Rev Rene. 2012;9(3):74-80.

14. Campos ALA, Araújo MAL, Melo SP, Gonçalves MLC. Epidemiologia da sífilis gestacional em Fortaleza, Ceará, Brasil: um agravo sem controle. Cad Saúde Pública. 2010;26(9):1747-55. https://doi. org/10.1590/S0102-311X2010000900008

15. Araújo EC, Costa KSG, Silva RS, Azevedo VNG, Lima FAZ. Importância do pré-natal na prevenção da sífilis congênita. Rev Para Med. 2006; 20(1):47-51. https://doi.org/10.5123/S0101-59072006000100008

16. Costa CC, Freitas LV, Sousa Nascimento DMN, Oliveira LL, Chagas ACMA, Lopes MVO, Damasceno AKC. Sífilis congênita no Ceará: análise epidemiológica de uma década. Rev Esc Enferm USP. 2013;47(1):152-9. https://doi.org/10.1590/S008062342013000100019

17. Araújo CL, Shimizu HE, Sousa AIA, Hamann EM. Incidência da sífilis congênita no Brasil e sua relação com a Estratégia Saúde da Família. Rev Saúde Pública. 2012;46(3):479-86. https://doi.org/10.1590/ S0034-89102012000300010 